\title{
Resonant seismic emission of subsurface objects
}

\author{
Valeri Korneev, Lawrence Berkeley National Laboratory
}

\begin{abstract}
The presented numerical modeling results and field data indicate that some contrast subsurface objects (such as tunnels, caves, pipes, filled pits, and fluid-filled fractures) are capable of generating durable resonant oscillations after trapping seismic energy. These oscillations are comprised of surface types of circumferential waves which repeatedly propagate around the object. The resonant emission of such trapped energy occurs primarily in the form of shear body waves that can be detected by remotely placed receivers. Resonant emission reveals itself in the form of sharp resonant peaks for the late parts of the records, when all the strong direct and primary reflected waves are gone. These peaks have been observed in the field data for a buried barrel filled with water, in 2D finite-difference modeling results and in the exact canonical solution for a fluid-filled sphere. A computed animation for the diffraction of a plane wave upon a low-velocity elastic sphere confirms the generation of resonances by durable surface waves. I show that resonant emission has characteristic quasi-hyperbolic travel-time patterns on shot-gathers. The inversion of these patterns can be performed in the frequency domain after muting the strong direct and primary scattered waves. Subsurface objects can be detected and imaged at a single resonance frequency without an accurate knowledge of source trigger time. The imaging of subsurface objects requires information about the shear velocity distribution in an embedding medium, which can be done interactively during inversion.
\end{abstract}




\section{INTRODUCTION}

Some contrast acoustic and elastic objects, such as tunnels (of different kinds), pipes, buried containers, UXOs, mine shafts, and fluid-filled fractures, are capable of resonating at certain frequencies as a result of trapping energy from passing waves and then slowly releasing that energy back into an outer medium. As high-contrast scatterers, these objects are capable of generating strong scattered waves in which primary (ballistic) PP, PS, SS waves carry away most of the energy brought in by the incident waves. This conclusion follows from numerical and analytical results obtained from the canonical solutions for spheres and cylinders (Frisk et al., 1975; Metsaveer, et al, 1979; Uberall, 1973; Veksler, 1980, 1984; Korneev and Johnson, 1996) and practically all of the scientific work done in determining underground scatterers rely on the utilization of these waves (e.g., Landa and Keydar, 1998; Groenenboom and Falk, 2000). Apart from the primary scattered waves, canonical solutions also predict the existence of other waves, which creep along the walls of the object while emitting secondary scattered energy into an embedding medium.

Waves that propagate around an object along its outer interface are called circumferential waves. The emissions coming from propagating circumferential waves have been intensively studied in hydro-acoustic problems (Frisk et al., 1975; Uberall, 1973; Veksler, 1980, 1984), and have two distinct features compared to a typical seismological application. Firstly, the embedding medium in hydro-acoustics is water, which is relatively homogeneous and conducts no shear waves. Secondly, in hydro-acoustic applications, both the source and the receiver are located at the same point, and therefore the recorded signals represent a back-scattering energy called echo-signals. These simplifying features, as well as the high practical importance of echo- 


\section{Resonant Seismic Emission}

locating problems, have resulted in thorough analytical and numerical studies of echo-signal compositions.

Four types of circumferential waves are known. The first type is the Frantz wave (Frantz, 1954), which propagates around the object with velocity approximately equal to that of the embedding medium. These waves merge with direct waves in the edges of the shadow zones and form most of the wave field in these zones (Groenenboom and van Dam, 2000). Frantz waves intensively radiate energy into the outer medium and rapidly decay in amplitude over time. The second type is comprised of "whispering gallery" waves (Rayleigh, 1924); they primarily propagate inside of an object being multiply reflected from a curved object's interface. Whispering gallery waves also decay rather rapidly because they lose their energy during reflections at interfaces. The third type of circumferential wave is the Rayleigh waves. Even if their initial amplitude is small compared to other waves, over a long observation time Rayleigh waves can dominate in records, as it happens with teleseismic events. Finally, the fourth type, Stoneley waves can be excited when either the object or the medium is a fluid (Uberall, 1973). Stoneley waves are also slowly decaying waves, and can make many propagation cycles around an object without significant attenuation.

For both high- and low-velocity objects, the primary scattered waves have the same order of magnitude. The main difference between these objects is in the later arrivals, when all the primary waves are already gone. While high-velocity objects effectively radiate most of their energy soon after an impact, low-velocity objects trap some fraction of the energy in the form of multiple reflections. High contrast objects of both types also support circumferential waves that slowly release their energy by radiating common body waves. The resonant character of echo signals was observed both in data and numerically. Sharp spectral peaks of these signals appear 
because of the slow amplitude decay of circumferential waves, where an object's geometry allows a constant rotation time around it.

Circumferential waves have been studied in an elastic outer medium, primarily for objects with cylindrical symmetry (Plona, et al., 1992; Hassan and Nagy, 1997). Applications include pipe testing and borehole condition analysis. In fluid-filled fractures with finite lengths, resonances can be supported by the slow Stoneley guided waves (Korneev, 2008). However, observations and numerical simulations of these resonances are yet to be developed. This paper demonstrates that resonant emission can be detected in both real and synthetic data. After simple processing, seismic emission records can be effectively used for seismic imaging and velocity estimations. The remarkable feature of resonant seismic emission is that it is predominantly comprised of shear body waves.

\section{FIELD EXPERIMENT}

\section{Experimental setup and data acquisition}

The field experiment (described in this paper), conducted in 1999, was intended to generate the test data set for subsurface object-detection and imaging methods. Soil for the experiment consisted of compacted, consolidated sand with a P-velocity of $500 \mathrm{~m} / \mathrm{s}$ and an S-velocity of 240 $\mathrm{m} / \mathrm{s}$. The object was a water-filled barrel $60 \mathrm{~cm}$ in diameter and $120 \mathrm{~cm}$ in height. Some air bubbles were left inside of the barrel before it was sealed. The barrel was placed at a depth of 5$6 \mathrm{~m}$ in a specially excavated pit, which was then back-filled with soil. A 24-channel $0.5 \mathrm{~m}$ spaced line of vertical geophones was used for every ground shot made by a sledge-hammer, with a shot point positioned at the first receiver of each line (Figure 1). There were 87 shots in total at a $1 \mathrm{~m}$ spacing interval. The middle receiver of Line 51 was directly above the barrel. The 


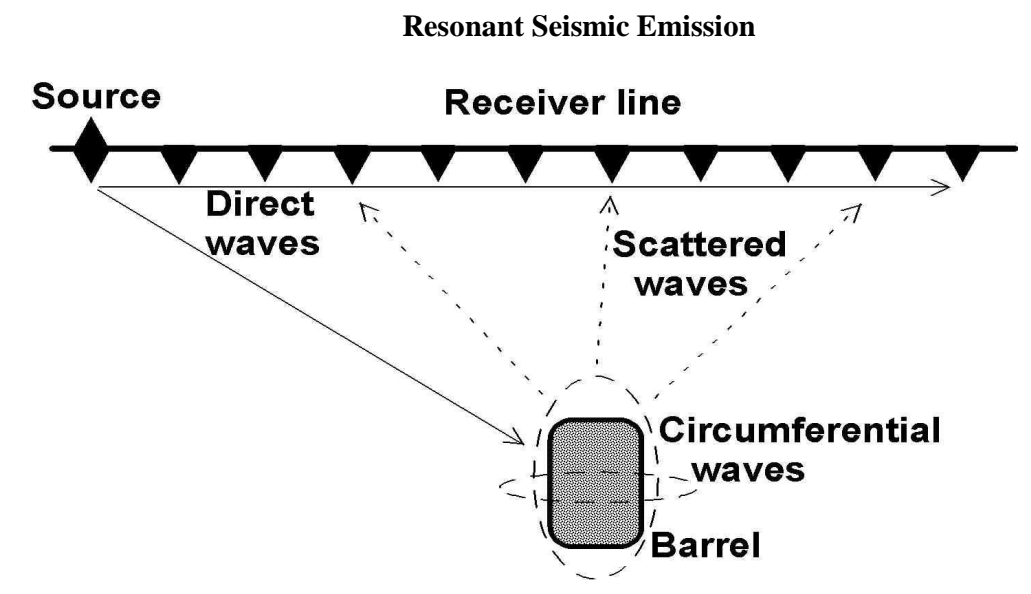

Figure 1. Geometry of the seismic experiment to locate a buried water-filled barrel. Solid, dashed and dotted line arrows indicate direct, circumferential and scattered waves respectively.

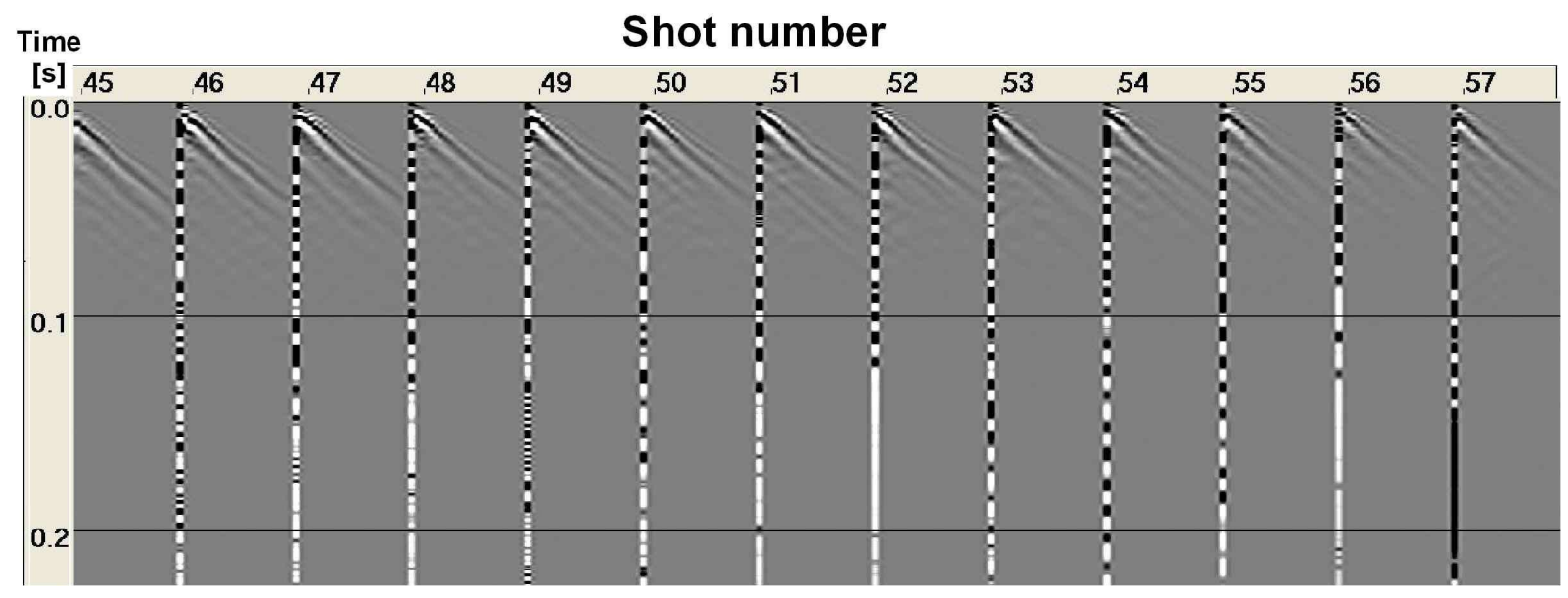

Figure 2. Shot gathers 45-57 for field data recorded above the buried barrel. Visible is the direct S-wave.

Barrel is located in the middle of gather 51. Contrast black-white striped lines correspond to the first receivers for each shot which are coincident with source positions.

records were 0.5 seconds in length with a $0.5 \mathrm{~ms}$ data sampling rate. Figure 2 shows raw shot gathers 45-57. Direct P- and S-waves have dominating amplitudes on each trace, and from 0.1 seconds after first arrivals, no coherent waves were visible in the data (Korneev and Landa, 2007). No primary scattered waves are visible in the background of the direct waves. (Some 


\section{Resonant Seismic Emission}

waves can be interpreted as reflections from the pit.) The author is unaware of any successful attempts to detect and image the barrel using these data.

\section{Imaging}

After applying automatic gain control (AGC) with a $25 \mathrm{~ms}$ window, the late $(0.25-0.5 \mathrm{~ms})$ interval revealed sharp resonant peaks in amplitude spectra at frequency $f_{\text {res }}=78 \mathrm{~Hz}$ (Figure 3).

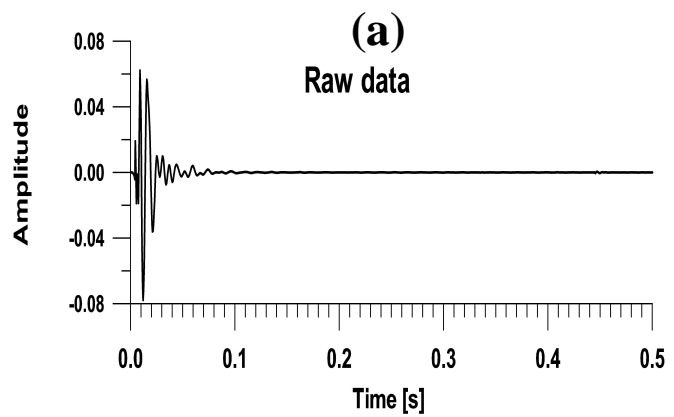

(c)

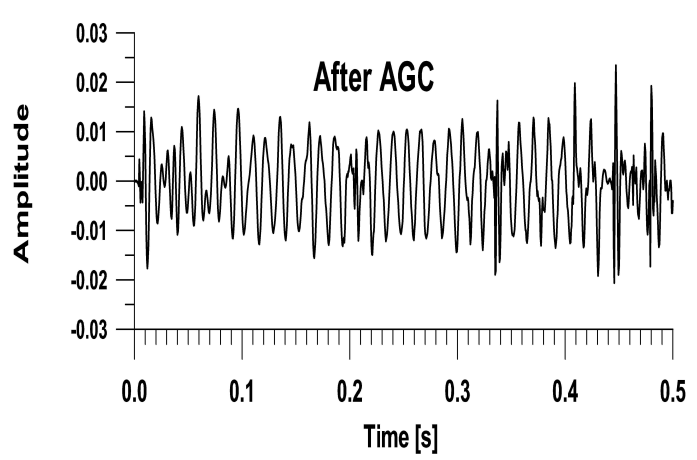

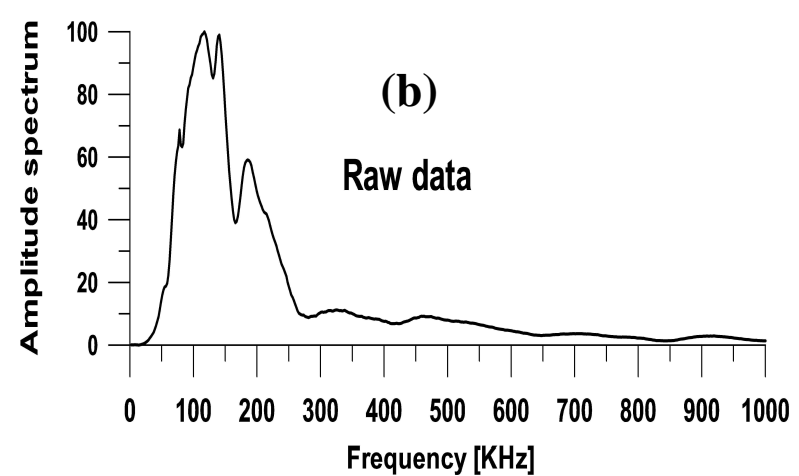

(d)

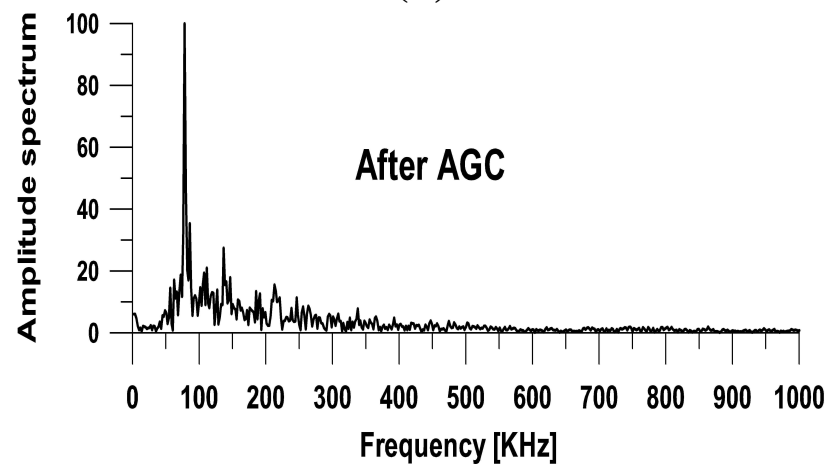

Figure 3. a) Single seismic trace recorded at surface over a buried water-filled barrel. b) Amplitude spectrum of trace in a). c) Result of applying AGC to trace in a). d) Amplitude spectrum of trace in c). Durable oscillation in late arrivals primarily consists of single harmonic at $78 \mathrm{~Hz}$.Band-pass filtering of traces in the $75-83 \mathrm{~Hz}$ interval yielded the clear hyperbolic signature of a secondary seismic source with the apex in the middle of Line 51 (Figure 4). The phase slopes $(240 \mathrm{~m} / \mathrm{s})$ of the secondary source correspond to the velocity $\mathrm{V}_{\mathrm{S}}$ of shear waves in an embedding medium. 


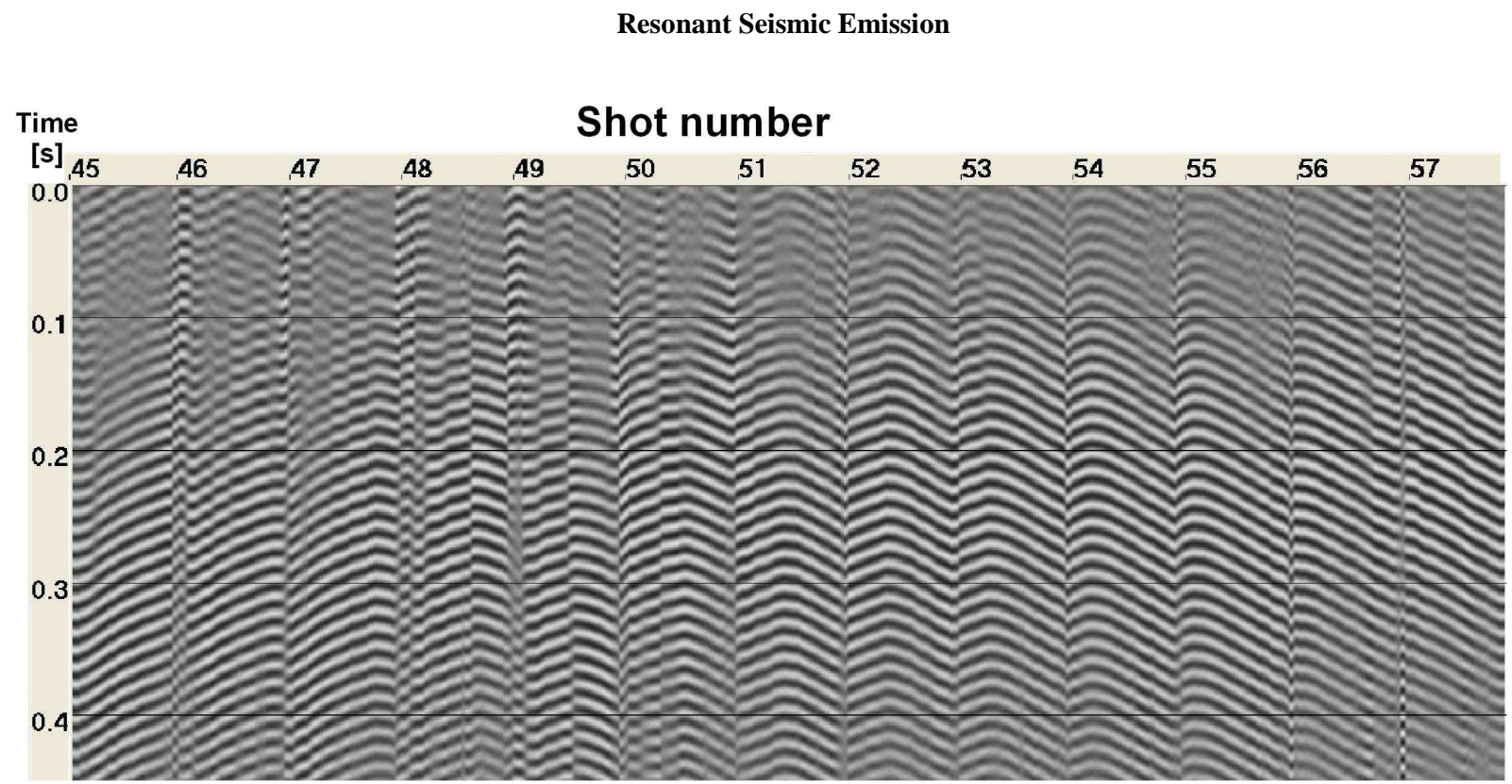

Figure 4. Shot gathers 45-57 after AGC and band-pass filtering around $78 \mathrm{~Hz}$ show hyperbolic monochromatic signatures of a secondary source. Hyperbola's apex indicates lateral position of the object while its curvature has direct relationship with object's depth.

If a velocity is known, single shot information is enough to locate the barrel. The apex location points at the horizontal coordinate, while fitting the hyperbolic curvature allows us to determine the barrel's depth. Utilizing information from all available data, we consider modeling the Green's function $W_{n k}(f)$ in the form

$$
W_{n k}\left(f_{r e s}\right)=\exp \left(i 2 \pi f_{r e s} \tau_{n k}\right) / r_{n k} \text {, }
$$

which describes the monochromatic wavefield excited by an underground secondary source at the point $M_{k} \equiv M_{k}\left(x_{k}, z_{k}\right)$ with spatial coordinates $x_{k}, z_{k}$, and record at the sensor location $S_{n} \equiv S_{n}\left(x_{n}, z_{n}\right)$, where $r_{n k}$ is the wave travel distance between points $M_{k}$ and $S_{n}$, and $i=\sqrt{-1}$.

The travel times $\tau_{n k}$ were computed assuming a homogeneous velocity

$$
\tau_{n k}=\frac{r_{n k}}{V_{S}}
$$

The imaging function was taken to be of the form 


$$
\begin{gathered}
\text { Resonant Seismic Emission } \\
F\left(M_{k}\right)=\sum_{j}\left|\sum_{n=1}^{N} s_{n j}{ }^{*}\left(f_{r e s}\right) W_{n k}\left(f_{r e s}\right)\right|,
\end{gathered}
$$

where $s_{n j}{ }^{*}\left(f_{\text {res }}\right)$ is the spectral component of data from shot $j$ recorded in receiver $n(1 \leq n \leq N)$ and contained in the 0.3-0.4 time interval from Figure 4. The star $(*)$ denotes complex conjugation. The summation in Equation 3 covers all the shots in the vicinity of the imaging area (shots 45-57). The use of absolute values in Equation 3 makes the inversion independent of shot origin times and stabilizes the inversion result, which is shown in Figure 5.

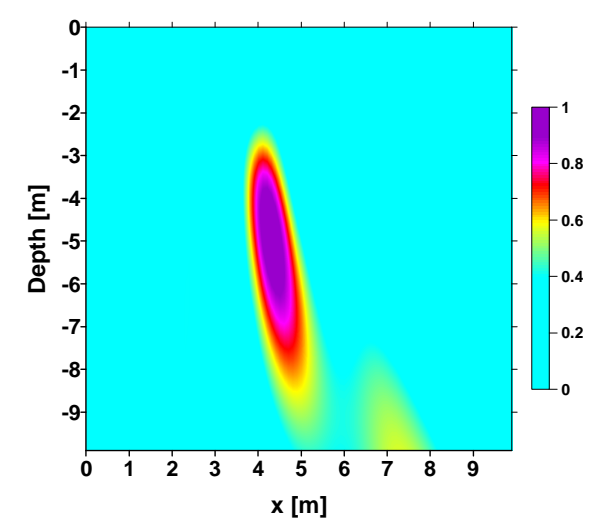

Figure 5. Migrated image of the buried barrel. Displayed is the function F from equation 3.

A similar inversion was performed in the time domain, but it required phase-picking, which is generally an unstable and non-unique procedure. The implementation of Equation 3 is simple, straightforward, and quick. The maximum value zone in Figure 5 indicates the barrel's correct location and contains contributions from all the used shots. To ensure the best image quality, the inversion was performed for different velocities $V_{S}$ in the $200-300 \mathrm{~m} / \mathrm{s}$ range. At incorrect velocities, the inversion image smears and splits in several anomalies. The best focusing was obtained for $V_{S}=235 \mathrm{~m} / \mathrm{s}$. Thus, the described imaging approach enables the evaluation of imaging velocities. 


\section{Modeling}

We modeled the field data with a $2 \mathrm{D}$ elastic finite-difference code, using a $40 \times 12.5 \mathrm{~m}$ model with $0.05 \mathrm{~m}$ grid spacing.

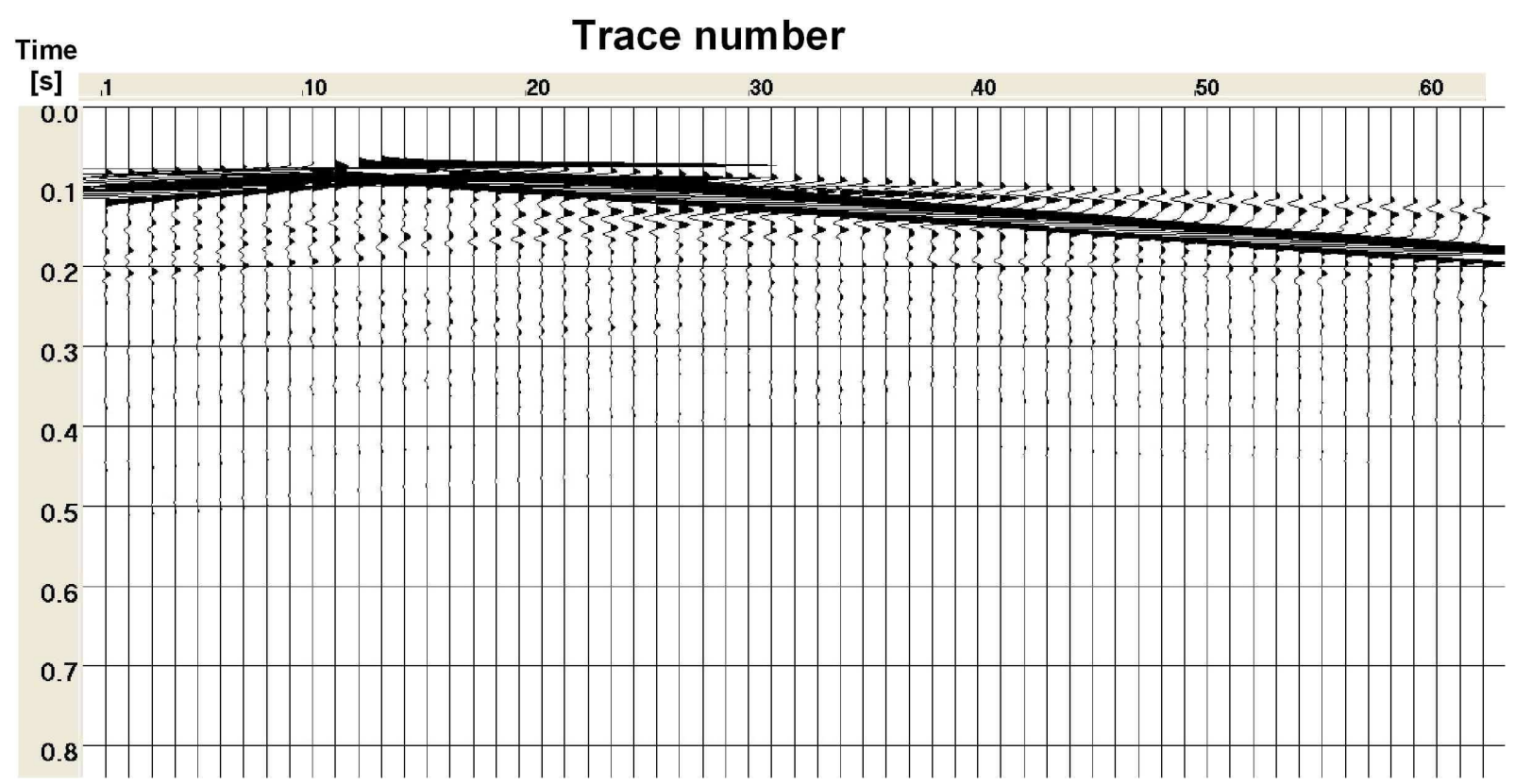

Figure 6. Single shot gather 3 for vertical component of the modeled traces. Amplitudes rapidly decay after first arriving phases.

\section{Circumferential} waves

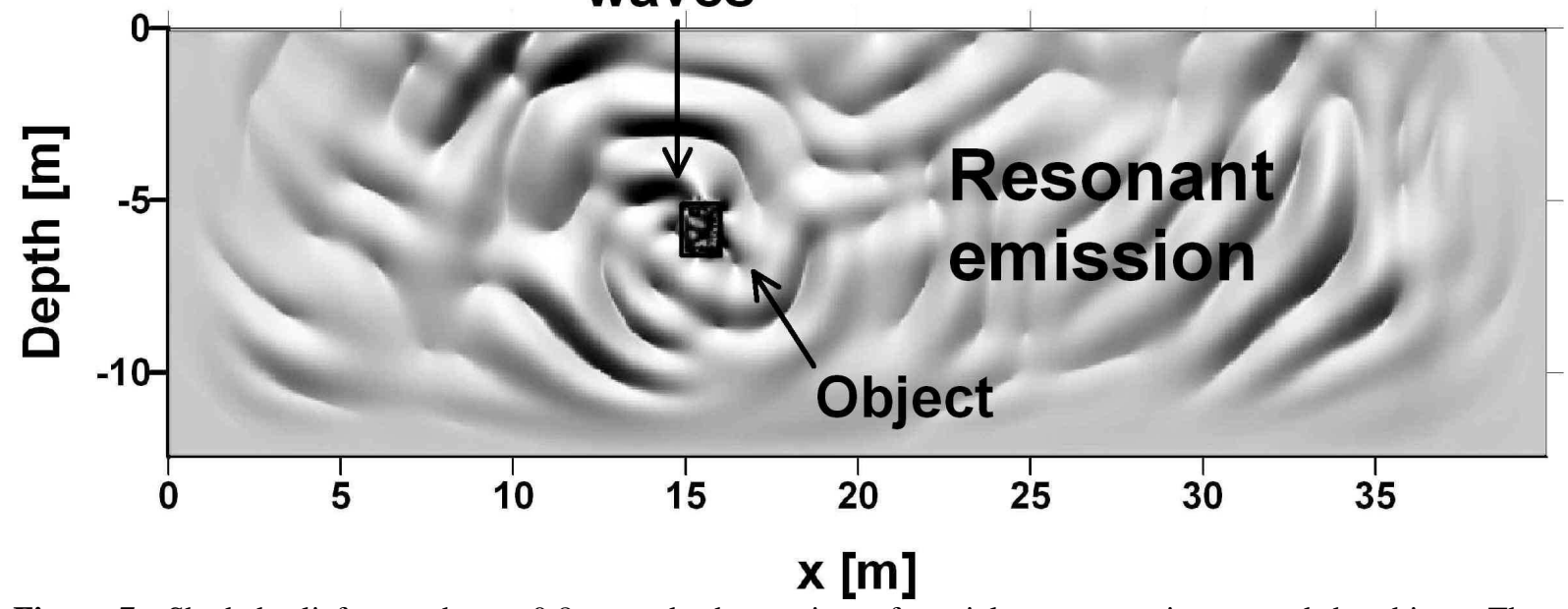

Figure 7. Shaded relief snap-shot at 0.8 seconds shows circumferential waves rotating around the object. These waves cause resonant emission (waves) propagating in embedding medium. 
(a)

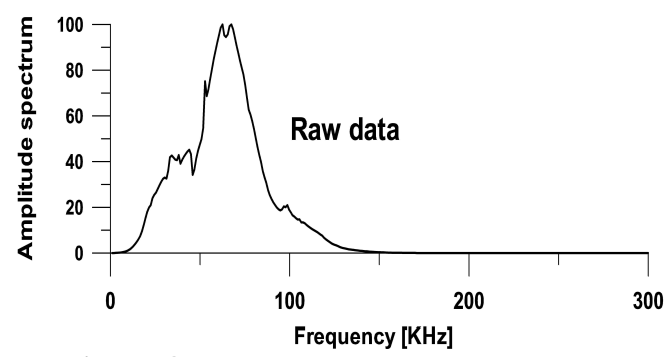

(b)

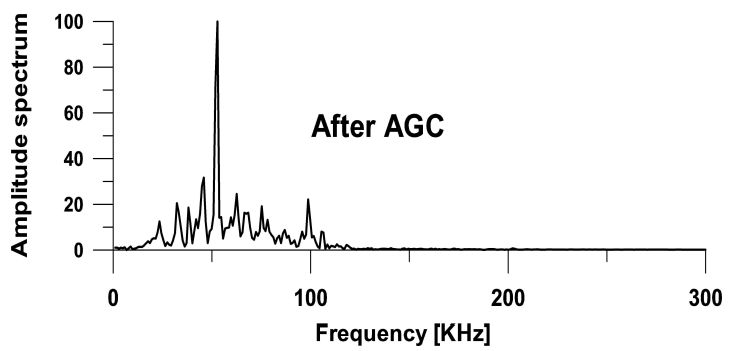

Figure 8. a) Amplitude spectrum of trace 30 from the shot-gather shown on Figure 6.

b) Amplitude spectrum of the same trace as on a) but after applying AGC reveals sharp peak at $53 \mathrm{~Hz}$ similarly to results for filed data from Figure 3.

The background Vp and Vs velocities had the same values as in the field experiment. The local object was modeled as a $1 \mathrm{~m} \times 0.7 \mathrm{~m}$ rectangular inhomogeneity containing fluid with $70 \mathrm{~m} / \mathrm{s}$ velocity and $1 \mathrm{~g} / \mathrm{cm}^{3}$ density. Ten shot gathers were computed using $3 \mathrm{~m}$ spacing for sources.

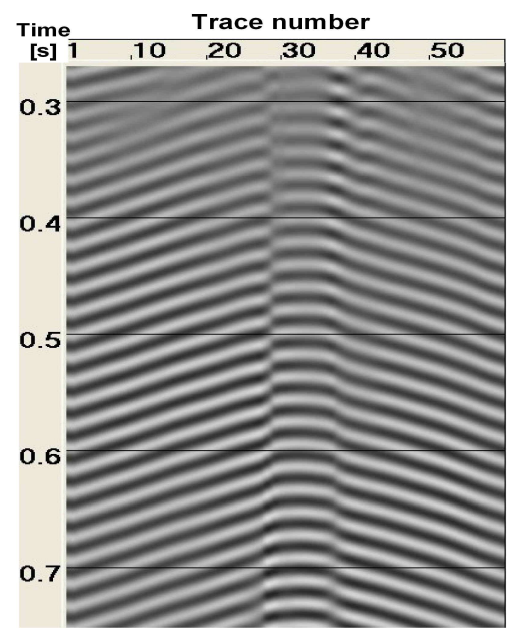

Figure 9. Late arrivals of modeled data after applying AGC and band-pass filtering around the resonance frequency have repeating quasi-hyperbolic pattern similar to one observed for field data (Figure 4).

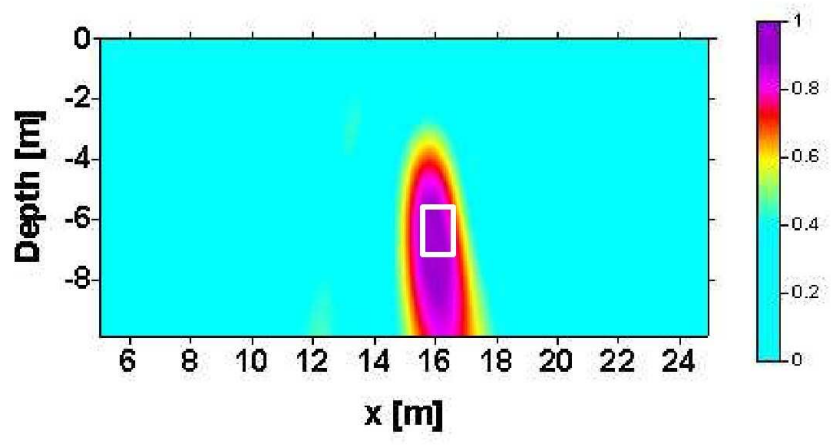

Figure 10. Migrated image of the modeled data locates the object and is similar to one obtained from field data. (Figure 5).

The surface receiver line was the same for all shots, and had 61 sensors separated by $0.5 \mathrm{~m}$. A single shot gather for Shot 3 is shown in Figure 6, where (similarly to the field case) the primary 
scattered waves rapidly decay after $0.2 \mathrm{~s}$. The recording length was $1 \mathrm{~s}$. A snapshot image at 0.3 s (Figure 7) shows that at a late propagating time, the field predominantly consists of shear waves created by circumferential waves in the object. The spectral content of the later parts of the records (after $0.5 \mathrm{~s}$ ) shows a sharp peak at $53 \mathrm{~Hz}$ in all the traces (Figure 8).

All the data were AGC'd and band-pass filtered around the peak frequency, leading to the images shown in Figure 9 and revealing quasi-hyperbolic phases similar to the ones observed in the real data (Figure 4). The application of the inversion procedure described in the previous section revealed a clear image of the heterogeneity (Figure 10).

\section{NUMERICAL EXPERIMENT: RESONANT SPHERE}

\section{General considerations}

Spherical models allow for analytical scattering solutions and therefore can be helpful in understanding the basic properties of the scattered fields formed on less treatable objects. Following the general formulation for diffraction upon a sphere (Korneev and Johnson, 1996), we present here some numerical results that illustrate the resonant scattering mechanism. An integral scattering characteristic called the "normalized scattering cross-section," $\sigma_{W},(W=P, S)$, relates the total energy of the scattered field to the energy of a P- or S- wave incident upon the object. For an elastic embedding medium, partial P- and S- scattering cross sections $\sigma_{P W}$ and $\sigma_{S W},(W=P, S)$ can be considered, which correspond to separate contributions of compressional and shear scattered waves. It has been shown (Korneev and Johnson, 1996) that for any spherical object,

$$
\frac{\sigma_{P S}}{\sigma_{S P}}=2 \frac{V_{P}^{4}}{V_{S}^{4}},
$$


and the seismic energy primarily converts into shear waves. In Equation (4), the velocities on the right-hand side correspond to the embedding medium.

\section{Results}

Figure 11 shows $\sigma_{P P}$ and $\sigma_{P S}$ partial scattering cross sections for a fluid filled sphere. The sphere has a radius of $\mathrm{R}=0.5 \mathrm{~m}$, and the fluid is characterized by $V_{F}=110 \mathrm{~m} / \mathrm{s}$ and a density of 1 $\mathrm{g} / \mathrm{cm}^{3}$.

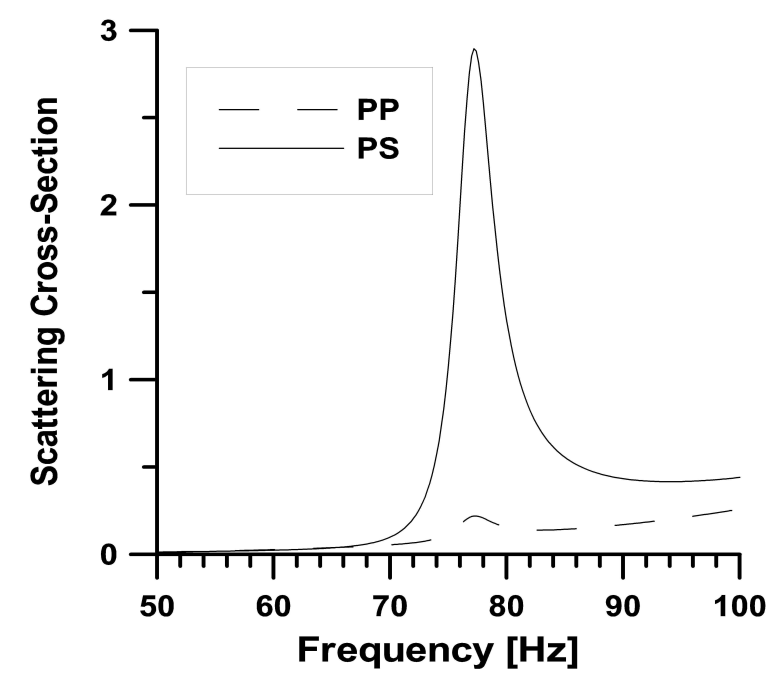

Figure 11. Scattering cross-section for fluid-filled sphere with resonant peak at $78 \mathrm{~Hz}$.

This model reflects the case in which the liquid contains some gas bubbles; such a mixture has the compressibility of gas and the density of liquid. Such a parameter combination leads to very low propagation velocities (Kaelin, 1998), which are lower than those in the embedding shallow subsurface. The chosen parameter set shows a sharp resonant peak at $78 \mathrm{~Hz}$, which coincides with the peak in real data (Figure 3). For both P- and S- types of incident waves, the scattering energy of S- waves overwhelms the energy of P- waves, and apparently can be neglected in real 


\section{Resonant Seismic Emission}

applications. The first resonant peaks for a fluid-filled sphere follow the equation (Korneev and Johnson, 1996)

$$
j_{2}(\xi)-\frac{j_{1}(\xi)}{\xi}=0, \quad \xi=\frac{\omega R}{V_{F}}
$$

containing the spherical Bessel functions $j_{k}(\xi)$ and the frequency $\omega$. The first three roots of this equation are $\xi \approx 2.1,5.9,9.2$. Thus, if resonance frequency is measured and velocity $V_{F}$ is known, the radius of the sphere $R$ can be determined using equation 5 .

\section{Animation}

A solution for the sphere was also used to compute an animation of plane P-wave diffraction upon an elastic low-velocity sphere. This sphere has a $1 \mathrm{~m}$ radius, a material with $\mathrm{Vp}=4500 \mathrm{~m} / \mathrm{s}$, Vs of $2600 \mathrm{~m} / \mathrm{s}$, and a density of $2.3 \mathrm{~g} / \mathrm{cm}^{3}$. The embedding medium has corresponding parameters $\mathrm{Vp}=6000 \mathrm{~m} / \mathrm{s}, \mathrm{Vs}=3500 \mathrm{~m} / \mathrm{s}$, and density $=2.7 \mathrm{~g} / \mathrm{cm}^{3}$. The $6 \mathrm{~m} \times 6 \mathrm{~m}$ frame was filled with a grid of pixels with $2 \mathrm{~cm}$ spacing. For each pixel, a $0.256 \mathrm{~s}$ long trace was computed by Fourier-transforming an analytical solution formulated in a frequency domain.

(a)

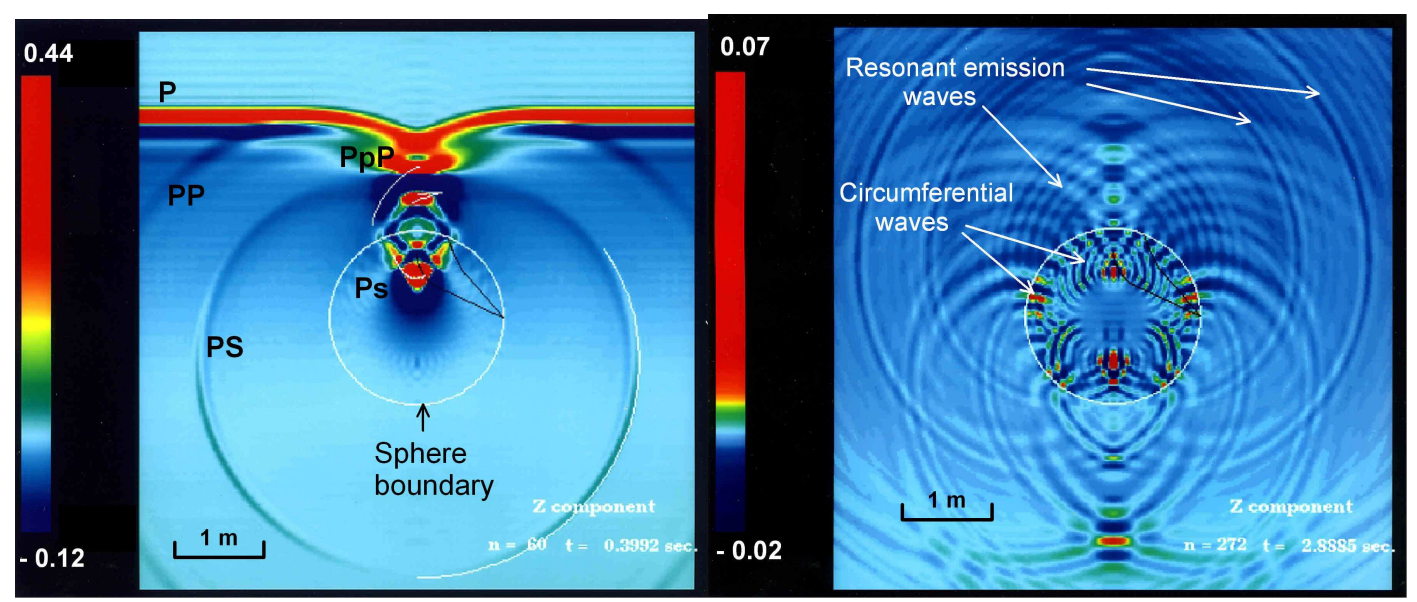

Figure 12. Snap-shots of field z-component for the low-velocity elastic sphere at $0.001 \mathrm{~ms}$ (a) when just primary scattered waves are formed, and at $0.02 \mathrm{~ms}$ (b) when just circumferential (Rayleigh) waves dominate the wavefield. 
Two time slices of the Z- component are shown in Figure 12 for $t 1=0.001 \mathrm{~s}(\mathrm{a})$ and $\mathrm{t} 2=0.02 \mathrm{~s}$ (b). The animation is computed for a P-plane incident wave. No assumptions about the boundaries other than sphericity are used. At the early time (a), the wavefield consists of geometrically interpretable reflected and refracted waves; at the late time (b), the dominant feature represents Rayleigh circumferential waves. Note that shorter S- waves penetrate deeper into the sphere while $\mathrm{P}$ - waves propagate close to the sphere interface. To maintain a dynamic resolution of the frames a variable color scale was applied. Note the regular "heartbeat" of the sphere at late times. These oscillations correspond to resonances caused by circumferential waves.

\section{DISCUSSION}

The imaging of shallow subsurface heterogeneities has a variety of important applications including the detection and location of seismically contrast objects. The primary scattered waves from such target objects may be used for object imaging; however, these waves closely interfere with other strong waves, making such imaging difficult. Resonant emission waves have relatively small amplitudes, but they are much more durable than primary scattered waves. The extraction of the resonant emission waves from late arrivals makes it possible to detect and image subsurface objects, using data at resonance frequencies. Resonant emission propagates in the embedding media primarily in the form of shear body waves, which is distinct from the hydro-acoustic case. This follows from the canonical solutions, reflecting the fact S- waves are slower than P-waves and that wave energy preferably converts into slow-velocity waves.

This imaging of resonating objects implements wave-migration ideas in a frequency domain. It uses the assumption that every underground location potentially contains a monochromatic source radiating seismic waves. In elastic media these radiated waves are mostly comprised of shear waves. The possibility of neglecting P- waves for inversion purposes makes 
imaging simple, robust, and practically insensitive to the size and extent of the data selection window. No data picking is necessary for inversion. In the case of several objects with different resonating frequencies, they can be imaged consecutively at each frequency. Knowledge about shear wave velocity in the embedding medium seems essential for a successful inversion. However, the relatively fast (seconds) inversion computation makes it possible to perform this inversion for a range of reasonable velocity distributions. A correct velocity distribution provides the sharpest localized image.

In practice, there are several classes of objects that contain elements with contrast velocities that are significantly different than those in the embedding media (tunnels, pipes, mine shafts, and cavities filled with gas, ice lenses, metallic objects, UXOs etc.). The high contrasts of such structures result in durable circumferential waves (primarily of the Stoneley type in fluid-filled, and of the Rayleigh type in elastic structures). Another class of wave trapping objects are the localized low-velocity zones which have naturally low velocity (as part of a coal seam) or which result from some impact, such as filled excavation pits, where loosened rock/soil has smaller elastic modulii compared with the embedding medium. The intrinsic attenuation inside of an object is a likely contributor to the decrease rate of wave amplitude.

A single object can have a set of resonant frequencies. The values of resonant frequencies depend on the object's size and material properties. When constraints are imposed on some of the object parameters, the resonant frequencies can be inverted for the remaining parameters, using analytical solutions, numerical modeling or calibration measurements. Indeed, if the object has a closed geometry enabling the surface wave to return to its original position after full rotation around the object, then the resonant period is simply the time difference between two consecutive rotations and it stays constant during the whole process. This period is defined by 


\section{Resonant Seismic Emission}

the length of the object's perimeter and the propagation velocity, and can be evaluated using the canonical wave propagation solutions for an appropriate geometry. Knowledge about the resonance frequency and the circumferential wave velocity enables the estimation of the perimeter length, giving the object's size for known shapes in a similar manner to the spherical example. Another important characteristic of resonant emission is the width of the resonant peak and(?) the decay rate in the time domain. Both phenomena describe resonant emission attenuation that likely depends on the material parameters of both an object and an embedding medium, as well as on the shape of the object's interface. The detailed study of such dependences is beyond the scope of this paper.

As for any new imaging approach, it should be considered for reservoir imaging applications. If the reservoir consists of flat sedimentary layers then only reflected waves can be recorded from them. Multiple reflections from such structures (although very periodic in time) rapidly decay in amplitude making their detection at late times nearly impossible. However, some structural hydrocarbon traps with local heterogeneities can possibly develop resonances and be imaged using the method described in this paper.

Fluid-filled fractures also can resonate and therefore be imaged using resonant emission. As it is recently shown in Korneev (2008), Stoneley guided waves for realistic fracture and fluid parameters have very low velocities and a large enough attenuation factor $Q$ to be able to propagate many wavelengths without significant attenuation. In particular, a $1 \mathrm{~mm}$ thick water/oil filled fracture resonates at frequency as low as $10 \mathrm{~Hz}$ when the fracture is just $4 \mathrm{~m}$ long. Stoneley guided waves do not radiate body waves during propagation until they scatter on fracture tips, which become the origins of secondary sources and generate body waves, which can potentially be used for fracture imaging. Such radiation was detected and explained (Chouet, 


\section{Resonant Seismic Emission}

1986; Ferrazzini and Aki, 1987) as being caused by the oscillation of volcanic molten lava. This oscillation can be excited by fracture propagation or by microseismic or teleseismic events. The monitoring of these oscillations should reveal information about the fracture dynamic and growth since the resonance frequencies and the location of radiation points change together with fracture geometry changes.

\section{CONCLUSIONS}

Low-velocity subsurface heterogeneities trap seismic energy and can release it long after the recordings of primary scattered waves. Trapped energy primarily consists of circumferential waves propagating along the perimeter of an object, and radiates in the surrounding medium as body waves. Circumferential waves propagate in both directions around an object, which potentially can create discontinuous travel-time curves. Most of the energy radiated back to the embedding medium is carried by shear waves. This simple data pattern makes it an attractive candidate for inversion techniques based on migration (back-propagation) principles. Unlike conventional diffraction imaging techniques, resonant emission imaging can be performed at a single frequency and does not require accurate information about source position. There is also no need to engage in laborious wave-separation pre-processing, because all the primary waves can be simply muted.

We expect that multiple objects can be detected and imaged using separation in the frequency domain. Moreover, objects with the same resonant frequencies should be possible to resolve spatially if they have distinctly different phase functions in receiver locations. Limits of the spatial resolution are defined by imaging wavelength similarly to common optical images. Clearly, the exact timing of a source excitation is not important: trapped energy radiation can possibly be observed in the presence of background noise, leading to cost-effective object 


\section{Resonant Seismic Emission}

detection techniques. Requirements on the sensor coverage are yet to be estimated. Overall, our new object detection method shows a good correspondence between field and modeled data. In its current formulation, the method is ready for extensive testing and tuning.

\section{ACKNOWLEDGMENTS}

This work was supported by the National Energy Technology Laboratory, Office of Fossil Energy Sciences, of the U.S. Department of Energy under Contract No. DE-AC02-05CH11231. Field data were provided by Eugeny Landa (OPERA); GeoKinetiK, LLC provided KinetiK Professional processing software.

\section{REFERENCES}

Chouet, B., 1986, Dynamics of a fluid-driven crack in three dimensions by the finite-difference method: Journal of Geophysical Research., 91 , 13967-13992.

Ferrazzini, V., and K. Aki, 1987, Slow waves trapped in a fluid-filled infinite crack: Implications for volcanic tremor, Journal of Geophysical Research, 92, 9215-9223.

Franz, W., 1954, Uber die greenshen Functionen des Zylinders und der Kugel: Z. Naturforschung, 9a, 705-716.

Frisk, G.V., J. W. Dickey, and H. Uberall, 1975, Surface wave modes on elastic cylinders: Journal of Acoustical Society of America, 58, no. 5, 996-1008.

Groenenboom, J., and J. Falk, 2000, Scattering by hydraulic fractures: Finite-difference modeling and laboratory data: Geophysics, 65, 612-622.

Groenenboom, J., and D. B. van Dam, 2000, Monitoring hydraulic fracture growth: Laboratory experiments: Geophysics, 65, no.2, 603-611.

Hassan, W., and P.B. Nagy, 1997, Circumferential creeping waves around a fluid-filled cylindrical cavity in an elastic medium: Journal of Acoustical Society of America, 101, no.5, 2496-2503.

Hickling, R., 1964, Analysis of echoes from solid elastic sphere in water: Journal of Acoustical Society of America, 36, no.6, 1124-1137. 
Kaelin, B., 1998, Seismic imaging of the shallow subsurface with high-frequency seismic measurements: Ph.D. Thesis, UC Berkeley.

Korneev, V.A, 2008, Slow waves in fractures filled with viscous fluid: Geophysics, 73, no1, 1-7.

Korneev, V. A., and L. R. Johnson, 1996, Scattering of P and S waves by spherically symmetric inclusion: in Pure and Applied Geophysics, 147, no.4, 675-718.

Korneev, V. A., and E. Landa, 2007, Imaging of subsurface objects using resonant seismic scattering, $77^{\text {th }}$ Annual International Meeting, SEG, 1113-1117.

Landa, E., and S. Keydar, 1998, Seismic monitoring of diffraction images for detection of local heterogeneities: Geophysics, 63, 1093-1100.

Metsaveer, Y.A., N. D. Veksler, and A.S. Stulov, 1979, Diffraction of acoustic pulses on elastic bodies: (In Russian), Moscow, Nauka.

Plona, T. J., B. K. Sinha, S. Kostek, and S. K. Chang, 1992, Axisymmetrical Wave-Propagation in Fluid-Loaded Cylindrical- Shells. 2. Theory Versus Experiment: Journal of the Acoustical Society of America, 92, no. 2, 1144-1155.

Rayleigh, L., 1924, The problem of whispering gallery: Philosophical Magazine, 27, 100- 115.

Uberall, N, 1973, Surface waves in acoustics: in Physical Acoustics, ed. by M.P. Mason and P.N.Thornston, N.Y., Academic Press, 10, 1-60.

Veksler, N. D., 1984, Resonant scattering in hydro-acoustics, (In Russian), Tallinn, Valgus,

Veksler, N. D., 1980, Scattering of pulses on elastic cylinders, (In Russian), Tallinn, Valgus, 